\title{
Statistical behavior of electrical breakdown in insulating polymers
}

\author{
Kai Wu, ${ }^{1, a)}$ Yang Wang, ${ }^{1}$ Yonghong Cheng, ${ }^{1}$ L. A. Dissado, ${ }^{1,2}$ and Xiaojun Liu ${ }^{3}$ \\ ${ }^{1}$ State Key Laboratory of Electrical Insulation and Power Equipment, Xi'an Jiaotong University, \\ Xi'an 710049, China \\ ${ }^{2}$ Department of Engineering, University of Leicester, Leicester LE1 7RH, United Kingdom \\ ${ }^{3}$ Key Laboratory of Modern Acoustics, Nanjing University, Nanjing 210093, China
}

(Received 1 November 2009; accepted 30 January 2010; published online 31 March 2010)

\begin{abstract}
A simulation model to investigate the statistical property of electrical breakdown is presented based on the field-assisted percolation model for dielectric breakdown, by expressing the disorder in morphology as randomly distributed trap barriers. The effects of sample area and large defects on the statistical property of breakdown are also studied, and the statistical behavior of the lifetime is investigated by considering the stochastic development of deteriorated regions. In addition an equation describing the breakdown probability in homogeneous materials is derived from percolation theory, which we show fits well to the simulation data. The breakdown probability is expressed in terms of trap distribution, and thus provides a way to connect the disorder in polymer morphology with the stochastic nature of breakdown. () 2010 American Institute of Physics.

[doi:10.1063/1.3342468]
\end{abstract}

\section{INTRODUCTION}

The statistical characteristic of insulation breakdown reflects the failure rate and reliability of insulation, and is important for manufacture and asset management. It is well known that breakdown strength in polymers shows a much larger dispersion than in liquids and gases, ${ }^{1}$ and the large dispersion is usually considered due to the weak points. As some external weak points (e.g., the metal protrusions on electrode and contamination particles, etc.) exist in all the insulation systems of gases, liquids, and solids, the large dispersion of breakdown in polymers cannot be attributed to these external weak points. Therefore, the internal structure (or the morphology) of polymers may play an important role in determining the stochastic behavior of breakdown.

The Weibull distribution, ${ }^{2}$ which is based on the model of "weakest links," has been extensively used as an empirical law. This distribution is one of the extreme value distributions $^{3}$ without definite physical implications on a relationship between the breakdown statistics and the internal structure of insulating polymers. Only a few theoretical approaches ${ }^{3,4}$ have been carried out to assess its validity in polymer breakdown, especially in the practically important region of low failure probability. It has also been shown that many breakdown processes governed by an initiation stage, commonly observed in ramp-to-breakdown experiments, have breakdown strength statistics described by the Gumbel distribution, which is a different extreme value statistic. The two statistics differ in the form of the property distribution from which the extreme value is chosen, with the Weibull form being applicable to breakdown generating features that approach their extreme as a decaying power law, while the Gumbel form occurs when the extreme is approached as a decaying exponential. In addition to these theoretical questions as to the applicability of the Weibull distribution to

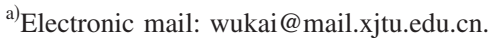

polymer breakdown, there are some experimental doubts as well, for example, it has been shown that the low field breakdowns measured with the large electrode deviated from the Weibull distribution. ${ }^{5,6}$ Furthermore the area effect of breakdown also showed deviation from the Weibull distribution (see also Ref. 3 for theoretical considerations of the same effect). In the two-parameter Weibull distribution, the scale parameter $\alpha$ and the shape parameter $\beta$ are fitted to experimental data as the following equation:

$$
P(E)=1-\exp \left[-\left(\frac{E}{\alpha}\right)^{\beta}\right],
$$

where $P(E)$ is the cumulative probability of failure for fields up to $E$.

If the sample area is enlarged to $n$-times, the failure probability can be written as

$$
P_{n}(E)=1-[1-P(E)]^{n}=1-\exp \left[-n\left(\frac{E}{\alpha}\right)^{\beta}\right] .
$$

Equation (2) indicates that if breakdown follows the Weibull distribution, the shape parameter $\beta$ should not change with the increase in electrode area and the value of $1 / \alpha^{\beta}$ should be proportional to the electrode area. However, these contradict with the experimental data of area dependence of breakdown. $^{5,6}$ The question of the applicability of the Weibull distribution to polymer breakdown is therefore still not resolved and models for the stochastic mechanism of breakdown are needed in order to study this question in more detail.

Recently, a percolation model for breakdown and aging has been set forward. ${ }^{7-9}$ This model describes the polymer as containing a three-dimensional cubic lattice of electron trap sites with a range of barriers for electron transfer between the sites. In the presence of an electric field the barrier heights are reduced and the sites with the barrier reduced to zero can be connected as clusters. When the electric field is high enough, a percolation cluster extending across the material 


\begin{tabular}{|c|c|}
\hline Network size & IVeibull Parameters \\
\hline $.200 \times 200 \times 200$ & $\alpha=0.952 \beta=42.1$ \\
\hline$\Delta 100 \times 100 \times 100$ & $\alpha=0.888 \quad \beta=17.9$ \\
\hline - $50 \times 50 \times 50$ & $\alpha=0.807 \beta=11.1$ \\
\hline$\times 30 \times 30 \times 30$ & $\alpha=0.778 \beta=6.55$ \\
\hline
\end{tabular}

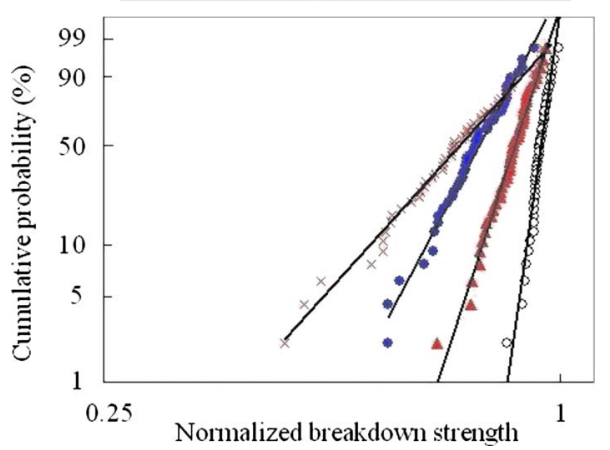

FIG. 1. (Color online) Weibull-distribution plot of breakdown in the samples with different sizes (each curve contains 50 simulated data).

appears to form an extended state in which electrons accelerate giving electrical breakdown. The Monte Carlo calculation has proven that a critical percolation threshold exists above which the percolation path appears. This is expressed through a parameter $\varphi(E)$, which defines the largest barrier that can be reduced to zero by an electric field of value $E$. For traps in which the trapped electron moves on a hydrogenic potential surface (Poole-Frenkel traps $\left.{ }^{10}\right), \varphi(E)$ can be written as

$$
\varphi(E)=\left(e^{3} / \pi \varepsilon\right)^{1 / 2} E^{1 / 2},
$$

where $\varepsilon$ is the permittivity. In this paper, we set forward an approach to study the stochastic behavior of breakdown on the basis of this model.

\section{SIMULATION AND ANALYSIS}

\section{A. Effects of sample size and defect distribution}

The field-assisted percolation model provides an approach to connect the stochastic behavior with the disorder in polymer structure. As polyethylene is a matrix containing a disordered disposition of the amorphous and crystalline phases and free volume (unoccupied lattice sites or nanoholes) it can be represented by a matrix of randomly distributed traps with the trap barriers in a certain range in the percolation model. ${ }^{8}$ However, because the percolation threshold is a constant and shows no dispersion in an infinite matrix, it is necessary to investigate the stochastic characteristics of breakdown in a matrix with limited size.

Figure 1 shows the simulated results in the cases of different network sizes. In Fig. 1, each curve is fitted to the data of 50 simulation samples. In each sample, the trap barrier at the sites of cubic network is assumed to be uniformly distributed in the range from 0 to $1 \mathrm{eV}$, and then the percolation threshold of the sample (i.e., the minimum field at which a percolation path connecting the upper and lower sides the network appears) can be calculated (refer to Ref. 7). Figure 1 shows that the simulated breakdown strength can be approximately fitted to a Weibull-distribution law. With the reduction in network size, the $\alpha$-value (i.e., the voltage at which

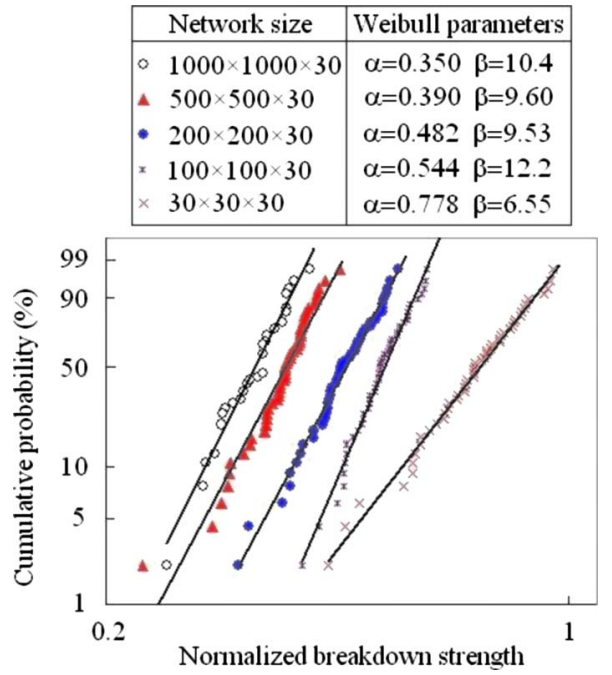

FIG. 2. (Color online) Weibull-distribution plot of breakdown in the samples with different areas (each curve contains 50 simulated data).

the accumulative probability of breakdown is $63 \%$ ) and the $\beta$-value decrease. When the network size is reduced from $100 \times 100 \times 100$ to $30 \times 30 \times 30$, the $\beta$-value becomes 6.55 from 17.9. For a trap of the Poole-Frenkel type, it has been shown that the interaction between trap barrier potentials is considerably reduced when the trap density is less than $10^{24}-10^{25} \mathrm{~m}^{-3}$, and thus the Coulombic force may affect a region with a radius of only about $10-20 \mathrm{~nm}$. However as the actual largest size of amorphous and crystalline phases and nanoholes may be over $100 \mathrm{~nm}$, the effective length of unit segment of the simulation matrix representative of the polymer may be significantly increased above that of the trap radius. Therefore the simulation results in Fig. 1 is very close to the experimental results that the $\beta$-value increased from less than 10 to over 20 when the sample thickness is increased from 4 to $14 \mu \mathrm{m} .{ }^{6}$ Here, in the following calculation, we chose the network thickness to be 30 for simplicity.

Figure 2 shows the simulated results in the cases of different sample areas when the trap barrier is assumed to be uniformly distributed in the range from 0 to $1 \mathrm{eV}$. Because of the edge effect of the sample, the $\beta$-value of small samples $(30 \times 30 \times 30)$ differs from those of the larger samples. When the sample area is larger than $200 \times 200$, the $\beta$-value seems not change with sample area, and the value of $1 / \alpha^{\beta}$ increases with the area (as Table I). These suggest that the Weibull distribution is a suitable statistical approximation. However, if a small concentration of large defects is introduced into the sample, the simulation results deviate from the Weibull distribution (as in Fig. 3). In Fig. 3, large defects are treated as traps with zero barrier ${ }^{8}$ (i.e., they are regions through which an electron can pass without hindrance, such

TABLE I. The value of $1 / \alpha^{\beta}$ at different sample areas.

\begin{tabular}{cc}
\hline \hline Sample area & $1 / \alpha^{\beta}$ \\
\hline $200 \times 200$ & 1048.5 \\
$500 \times 500$ & 8429.1 \\
$1000 \times 1000$ & 55168.2 \\
\hline \hline
\end{tabular}




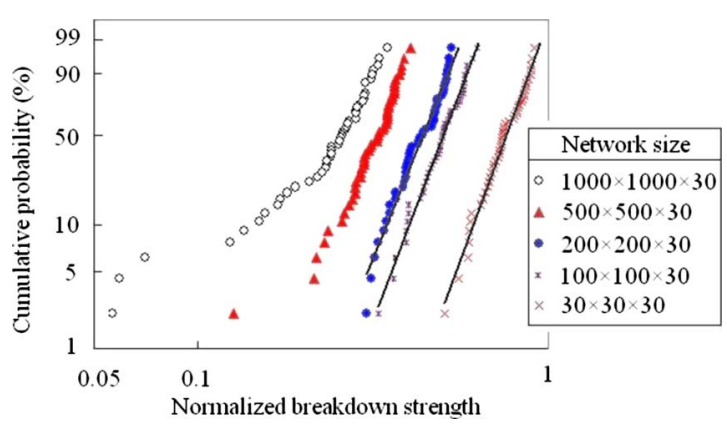

FIG. 3. (Color online) Weibull-distribution plot of breakdown in the samples with different areas and large defects (each curve contains 50 simulated data).

as material space unoccupied by polymer atoms) are assumed to be distributed randomly in the sample. The defect size and the density of defect number are assumed to be 12 $\times 12 \times 12$ (corresponding to a one micron diameter void when the unit segment in simulation network is $100 \mathrm{~nm}$ ) and $0.4 / 100^{3}$, respectively. Because of the small concentration of large defects, few of them exist in the small samples with limited number, and thus the simulated results of small samples still approximately follow the Weibull distribution. However, in the large samples, the results of low breakdown strength deviate from the Weibull distribution. These agrees with the experimental results of other authors. ${ }^{5,6}$ In practice, these large defects may be formed on the material surface or bulk due to thermal instability, mechanical vibration, or internal stress in the manufacture process.

The effect of large defects can also explain the phenomena that the $\beta$-value tends to be smaller in actual thick samples although it increases with sample thickness in the case of thin films. ${ }^{6}$ In fact, although the usual sample for breakdown test is about $1 \mathrm{~mm}$, the effective thickness to dominate breakdown may be much thinner than the sample thickness. Under dc voltage, because of the effect of space charge, the thickness of the layer with maximum field may be just about $1 \mu \mathrm{m}$. Under ac or impulse voltage, after the initial electron avalanche driven by the higher field develops in a certain distance, a high density of space charge can be formed, leading to the local field in front of the breakdown path high enough to make the breakdown self-sustainable. Thus, here, we suppose that the effective thickness to dominate initial breakdown may not be larger than decades of microns in thick samples, and the larger dispersion of breakdown (i.e., a smaller $\beta$-value) in usual thick samples can be explained in terms of the existence of large defects.

It should be pointed out that the actual breakdown strength is higher than the critical percolation threshold because a final breakdown occurs when the electron multiplication is developed to a certain value. The effects of electron multiplication or the thermal conduction of films lead to the thickness dependence of breakdown (i.e., the increase in breakdown strength with thickness reduction). ${ }^{10}$ This is different from the simulation results in Fig. 1 showing that the critical percolation threshold decreases with thickness reduction. Here, although the critical percolation threshold is different from the actual breakdown strength, we assume that its dispersion can reflect the stochastic property of actual break-

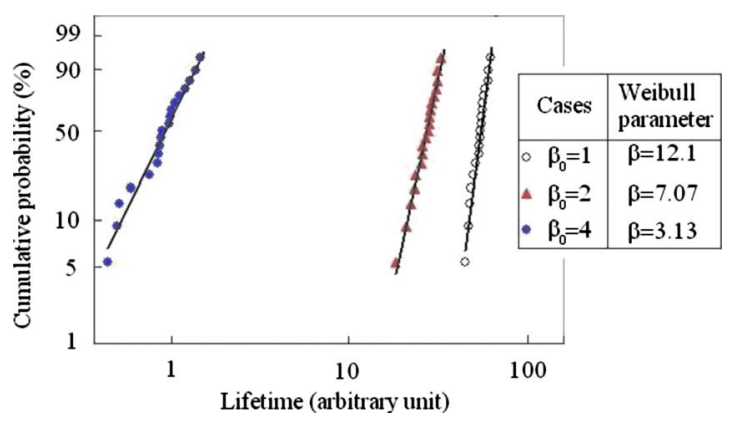

FIG. 4. (Color online) Weibull-distribution plot of lifetime (the sample size is $100 \times 100 \times 30$, each curve contains 20 simulated data).

down. In actual thick samples when the sample thickness is larger than the effective thickness to dominate initial breakdown, the critical percolation threshold should be quite close the actual breakdown strength.

\section{B. Stochastic property of life time}

By using the previous model for electrical aging, ${ }^{9}$ which described the development of deteriorated regions, the stochastic property of lifetime can be also investigated. Here, as in the previous model, ${ }^{9}$ we assume that $2 \%$ of the sites originally exist with zero barriers, and the deteriorated sites are also assumed to be of zero barrier. The local deterioration, i.e., the rate of one site changing to deteriorated sites due to the aging effect, is expressed as

$$
r_{i}=\sum_{j} f\left(E_{i j}, l_{j}\right),
$$

where the summation $\Sigma_{j}$ is taken for all the neighbor sites of site $i . f\left(E_{i j}, l_{j}\right)$ is a function standing for the contribution to the deterioration from the direction of the neighbor site $j . E_{i j}$ is the electrical field between the sites $i$ and $j$. Here, $f\left(E_{i j}, l_{j}\right)$ is not only related to field but also to the size of existing deteriorated region. This agrees with the simulation of aging by Dissado and Thabet, ${ }^{4}$ in which the extension of deterioration depended on the surrounding damage and deterioration size,

$$
f\left(E_{i j}, l_{j}\right) \propto\left\{\begin{array}{cl}
\left(E_{i j}\right)^{\alpha_{0}}\left(l_{j}\right)^{\beta_{0}}, & \left(l_{j} \geq 1\right) \\
\left(E_{i j}\right)^{\alpha_{0}}, & \left(l_{j}=0\right),
\end{array}\right.
$$

where $l_{j}$ is the maximum distance along the field from the edge of deteriorated region to a site $j$ inside the region, and $E$ is the field in the deteriorated region. $\alpha_{0}$ and $\beta_{0}$ are constants. $\beta_{0}$ influences the accumulation pattern of deteriorated region, percolationlike breakdown corresponds to $\beta_{0} \leq 2$, in which the percolation threshold is reduced due to the gradual global increase in deteriorated sites, and avalanchelike breakdown corresponds to $\beta_{0}>2$, in which breakdown occurs by the rapid growth of a filamentary structure. This latter behavior corresponds to that found in Ref. 4 where the deteriorated region was assumed to be conducting and hence giving an enhancement of the local electric field.

Here, in this paper, we define the lifetime as the time when the breakdown strength of a sample decreases to $1 / 10$ of the original average breakdown strength, and the lifetime of different samples are simulated (Fig. 4). Because $\alpha_{0}$ does 
not influence the accumulation pattern of deteriorated region, ${ }^{9}$ we choose $\alpha_{0}=2$ in our simulation. In order to save computation time, we choose the sample size to be 100 $\times 100 \times 30$, and 20 sample data are simulated in each case. Figure 4 shows that the lifetime can be still fitted to the Weibull distribution. Moreover, the slope of the fitted lines in Fig. 4 (i.e., the $\beta$-value) decreases with the increase in $\beta_{0}$, indicating the increase in lifetime dispersion with the increase in $\beta_{0}$. Because the stochastic property of lifetime is not only related to the disorder in morphology, but also related to the stochastic development of the deteriorated region, the avalanchelike breakdown leads to a larger dispersion of lifetime than percolationlike breakdown. The latter form of breakdown relates to a global state of deterioration and would tend to have a development that approaches toward deterministic behavior, which would have $\beta=\infty$. As actual $\beta$-values in aging life experiments are usually $0.5-2$, it is suggested that avalanchelike breakdown tends to occur in polymers, in which the deterioration rate is closely related to the size of deteriorated region, as found in Ref. 4.

\section{DISCUSSION}

Considering that the trap sites with barrier below $\varphi(E)$ may connect to form the percolation clusters at field $E$, the accumulative breakdown probability at field $E$ can be expressed as the probability of finding one cluster's length larger than a certain value $l$ (i.e., the sample thickness in our simulation). Although a similar assumption that breakdown occurs when the maximum cluster's length becomes large enough was proposed by other authors, ${ }^{10-12}$ here, a quantitative approach to describe the stochastic behavior of electrical breakdown strength and lifetime can be derived from our model. In a matrix with finite size, the probability of no clusters larger than $l$ can be written as ${ }^{13,14}$

$$
C(l)=\exp \left[-c L^{d} \exp (-k l)\right]
$$

where $c$ and $k$ are constants, $d$ is the dimension, $L$ is the sample size, and $L^{d}$ represents the sample volume.

Equation (6) is the appropriate distribution function far from the percolation threshold, ${ }^{14}$ and thus is suitable to describe the low field breakdown in our simulation. Because the percolation correlation length $\xi$ changes with the field $E$ [or the fraction of traps with barrier less than $\varphi(E)$ ], it is reasonable to assume that $C(l)$ should be approximately constant when $l=\xi$ at different fields. Thus Eq. (6) can be written as

$$
C(l)=\exp \left[-c L^{d} \exp \left(-k^{\prime} l / \xi\right)\right] .
$$

In the classical percolation theory, the correlation length $\xi$ follows the scaling law as ${ }^{15}$

$$
\xi \propto\left(p_{c}-p\right)^{-\nu},
$$

where $p$ is the fraction of conductive sites, $p_{c}$ is the percolation threshold, $\nu$ is a critical exponent, and is 0.85 for three dimensions

Therefore, if the trap barrier is distributed uniformly, the probability of no breakdown can be written as

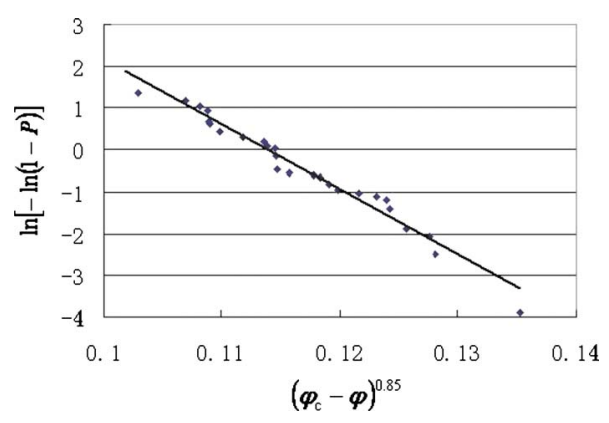

FIG. 5. (Color online) Plot of the data in the case of $1000 \times 1000 \times 30$ in Fig. 2 in the form of $\left(\varphi_{c}-\varphi\right)^{\nu}$ vs $\ln [-\ln (1-P)]$. The linear behavior of the data can be fitted to Eq. (7).

$$
C(E)=\exp \left\{-c S l \exp \left\{-k^{\prime} l\left[\varphi_{c}-\varphi(E)\right]^{\nu}\right\}\right\}
$$

where $S$ is the sample area.

In a sample with fixed volume, $S l_{0}$ is a constant. Also considering Eq. (3), the accumulative failure probability can be written as

$$
P(E)=1-C(E)=1-\exp \left[-A \exp \left\{B\left[\varphi_{c}-\varphi(E)\right]^{\nu}\right\}\right],
$$

where $A$ and $B$ are constants.

In the case of Poole-Frenkel-type traps with the barriers distributed uniformly, Eq. (10) can be written as

$$
P(E)=1-\exp \left\{-A \exp \left[B(D-\sqrt{E})^{\nu}\right]\right\} .
$$

Here $D$ is a constant.

Figure 5 confirms that the simulation data in Fig. 2 can be fitted to Eq. (10) very well. Here, Eq. (10) is different from the one derived for dielectric composites with conductive particles, ${ }^{13,14}$ in which the conductive particles may affect the field distribution. In our model of field-assisted percolation, we assume uniform field distribution in the sample, and thus this model is more suitable for homogeneous materials.

In the case where large defects (i.e., regions with zero barrier) are added with low concentration, the probability of no breakdown in the area containing the large defects can be written as

$$
C_{1}(E)=\exp \left\{-c a S\left(l-l_{d}\right) \exp \left\{-k^{\prime}\left(l-l_{d}\right)\left[\varphi_{c}-\varphi(E)\right]^{\nu}\right\}\right\},
$$

where $a$ and $l_{d}$ are the concentration and size of large defects, respectively.

Therefore, the total accumulative breakdown probability of the sample with large defects can be written as

$$
\begin{aligned}
P^{\prime}(E)= & 1-\exp \left\{-c(1-a) S l \exp \left\{-k^{\prime} l\left[\varphi_{c}-\varphi(E)\right]^{\nu}\right\}\right\} \\
& -\exp \left\{-c a S\left(l-l_{d}\right) \exp \left\{-k^{\prime}\left(l-l_{d}\right)\left[\varphi_{c}\right.\right.\right. \\
& \left.\left.-\varphi(E)]^{\nu}\right\}\right\} .
\end{aligned}
$$

Equation (13) can explain the simulation data in the cases of large sample areas in Fig. 3.

Although the Weibull-distribution law fits our data well, our simulation model and Eqs. (8)-(13) have definite physical meaning. They express the breakdown probability in 
terms of trap distribution, and thus provide an approach to connect the disorder in polymer morphology with the stochastic nature of breakdown.

\section{CONCLUSION}

By expressing the disorder in morphology as randomly distributed trap barriers, an approach to connect the disorder in polymer morphology with the stochastic nature of breakdown is set forward based on the field-assisted percolation model for dielectric breakdown, and an equation describing the breakdown probability in terms of trap distribution is derived. Assuming that one simulation segment represents an actual length of several decades to $100 \mathrm{~nm}$, the simulated $\beta$-value of the Weibull distribution in the sample with different thickness agrees very well with the experimental results of small-area samples with thickness ranging from 4 to $14 \mu \mathrm{m}$. The large dispersion of breakdown strength in usual samples is explained in terms of defect distribution and effective thickness to dominate initial breakdown. In aging process, the stochastic property of lifetime is not only related to the disorder in morphology but also related to the stochastic development of the deteriorated region.

${ }^{1}$ S. Okabe, N. Hayakawa, H. Murase, H. Hama, and H. Okubo, IEEE Trans. Dielectr. Electr. Insul. 13, 327 (2006).

${ }^{2}$ W. Weibull, J. Appl. Mech.-Trans. ASME 18, 293 (1951).

${ }^{3}$ L. A. Dissado, IEEE Trans. Dielectr. Electr. Insul. 9, 860 (2002).

${ }^{4}$ L. A. Dissado and A. Thabet, J. Phys. D: Appl. Phys. 41, 085412 (2008).

${ }^{5}$ S. J. Laihonen, A. Gustafsson, U. Gafvert, T. Schutte, and U. W. Gedde, IEEE Trans. Dielectr. Electr. Insul. 14, 263 (2007).

${ }^{6}$ S. J. Laihonen, U. Gafvert, T. Schutte, and U. W. Gedde, IEEE Trans. Dielectr. Electr. Insul. 14, 275 (2007).

${ }^{7}$ K. Wu, L. A. Dissado, and T. Okamoto, Appl. Phys. Lett. 85, 4454 (2004).

${ }^{8}$ K. Wu, T. Okamoto, and Y. Suzuoki, J. Appl. Phys. 98, 114102 (2005).

${ }^{9}$ K. Wu and Y. H. Cheng, J. Appl. Phys. 101, 064113 (2007).

${ }^{10}$ L. A. Dissado and J. C. Fothergill, Electrical Degradation and Breakdown in Polymers (Peregrinus, London, 1992).

${ }^{11}$ W. A. Curtin and H. Scher, Phys. Rev. B 55, 12038 (1997).

${ }^{12}$ M. I. Zeifman and D. Ingman, J. Appl. Phys. 88, 76 (2000).

${ }^{13}$ P. D. Beale and P. M. Duxbury, Phys. Rev. B 37, 2785 (1988)

${ }^{14}$ P. M. Duxbury and P. D. Beale, J. Phys. A 20, L411 (1987).

${ }^{15}$ R. Zallen, The Physics of Amorphous Solids (Wiley-Interscience, New York, 1983). 